\title{
The Advection Diffusion-in-Secondary Saturation Movement Equation and Its Application to Concentration Gradient-Driven Saturation Kinetic Flow
}

\author{
Tafireyi Nemaura ${ }^{1,2}$ \\ ${ }^{1}$ Department of Clinical Pharmacology, University of Zimbabwe, Harare, Zimbabwe \\ ${ }^{2}$ Department of Applied Mathematics, National University of Science and Technology, Bulawayo, Zimbabwe \\ Email: tnemaura@gmail.com, tafireyi.nemaura@nust.ac.zw
}

How to cite this paper: Nemaura, T. (2016) The Advection Diffusion-in-Secondary Saturation Movement Equation and Its Application to Concentration Gradient-Driven Saturation Kinetic Flow. Journal of Applied Mathematics and Physics, 4, 1998-2010. http://dx.doi.org/10.4236/jamp.2016.411200

Received: September 14, 2016 Accepted: November 11, 2016 Published: November 14, 2016

Copyright $\odot 2016$ by author and Scientific Research Publishing Inc. This work is licensed under the Creative Commons Attribution International License (CC BY 4.0).

http://creativecommons.org/licenses/by/4.0/

\begin{abstract}
This work describes the deterministic interaction of a diffusing particle of efavirenz through concentration gradient. Simulated pharmacokinetic data from patients on efavirenz are used. The Fourier's Equation is used to infer on transfer of movement between solution particles. The work investigates diffusion using Fick's analogy, but in a different variable space. Two important movement fluxes of a solution particle are derived an absorbing one identified as conductivity and a dispersing one identified as diffusivity. The Fourier's Equation can be used to describe the process of gain/loss of movement in formation of a solution particle in an individual.
\end{abstract}

\section{Keywords}

Partial Differential Equations, Conductivity Flux, Diffusivity Flux

\section{Introduction}

The derivation of an important equation by Fourier brought so many insights in the study of dynamics of flow through his law of thermal conduction [1]. It is an equation that has shaped the study of flow in liquids, solids and gases, for over two centuries. Additionally, Fourier proposed a method of solution to the equation using separation of variables [2]. The present work adopts similar construction arguments but differs in method of solution and variable space [3]. Fick used the same analogy of heat conduction in modelling diffusion [1]. Thus, Fick formulated what is now known as Fick's Second Law of Diffusion. Fick's work suggests that the diffusivity parameter is a con- 
stant [1] [4] [5]. The present work adopts a variable, concentration which enables the study of diffusivity/conductivity fluxes with the aid of movement gradient (observable from differences in concentrations) in a medium and time as independent variables instead of a location variable and time. It notes that the anti-solvation inducing measure (concentration) is also implicitly a function of time. The dependent variable is the secondary saturation movement which can be independently written as a function of time or concentration. Thus, the partial differential equation modelling movement derived from formulation reduces to an ordinary differential equation.

Further, the work notes that "diffusivity" is not independent of concentration as proposed by Fick's laws and proposes composition-dependent diffusion coefficients as suggested by Boltzmann, but differs in form [1] [4]. We propose a novel form of conductivity flux, a function of concentration, time, movement density and specific movement capacity. The motivation for modelling secondary saturation movement of solution particle in tracking diffusivity is that the variable increases with increasing concentration. However, the relationship of convection and advection with concentration is concave [6]. In addition, it is the only secondary movement measure that for a unique value of saturation movement there is a corresponding unique value of concentration. This results in less ambiguity in the formulation process. The derivation from the flow equation allows further understanding of diffusivity and conductivity fluxes of concentration in media.

Conductivity flux in this work defines the zero sum inward acquiring "concentration" movement of an interacting solution particle in a volume space from a neighbouring solution particle or central volume space in a solution particle bridge. A solution particle bridge is a state of exchange of concentration material between two interacting solution particles. Diffusivity flux is the zero sum outward dispersing concentration movement generated from an interacting solution particle to a neighbouring solution particle or peripheral volume space in a solution particle bridge. A total movement flux is postulated to be comprised of four main entities. These components have been identified as advective, saturation, passive and convective. Advective diffusivity is the deceleration of the advective diffusivity flux. Advective conductivity is the acceleration of advective conductivity flux.

The work considers definitions of important terms of advective movement flux. They are saturation kinetic advective conductivity and saturation kinetic advective diffusivity. Saturation kinetic advective conductivity flux is defined as the solution particle's inward formulation velocity rate constant of the secondary saturation movement with respect to advection. Additionally, saturation kinetic advective diffusivity flux as the interacting solution particle's outward formulation velocity of the secondary saturation movement of an interacting solution particle with respect to advection (rate of change of velocity rate constant of the volume $(\mathrm{ml})$ that dissolves a mass of $1 \mu \mathrm{g}$ of efavirenz with respect to advection). These two important parameters vary with the state and properties of the volumetric spaces (Central and Peripheral), the concentration of the solution particle and possibly the factors that affect absorption and elimination of solu- 
tion particle.

Diffusivity and conductivity fluxes can be tracked using the saturation relation that governs kinetic solubility movement in individuals to infer on the probable resultant effects. The solution particle characterisation is tracked in an individual through time and concentration. The work characterizes conductivity and diffusivity fluxes by studying the secondary saturation movement dynamics in solution particle's bridge. The calculations are done in the concentration-time space.

This work can further be developed to construct similar arguments for the primary saturation [6]. However, the primary level is not apparent. The primary saturation is a component of potential. On the other hand, the secondary saturation is the initial realized potential of solution-particle formation movement. Furthermore, the primary system is a sub-system of the secondary system.

\section{Methods}

Simulated projected data on secondary saturation movement, time and concentration was obtained from pharmacokinetic projections made on patients on $600 \mathrm{mg}$ dose of efavirenz considered in Nemaura (2014 \& 2015). Partial and Ordinary Differential equations are used in the development of models. A statistical Package $\mathrm{R}$ is used to develop nonlinear regression models.

\section{Derivation of Advection Kinetic Flow for the Secondary Saturation Movement}

Let $F(x, t)(/ h)$ be the secondary saturation movement at time $t$ and concentration $x$ (Gradient-driven transportation inducing measure) in the blood. The variable $F(x, t)$, is a measurement of density of movement. There is derivation of the flow equation of $F(x, t)$ from two physical "laws", that are assumed to be valid:

- The amount of movement required to raise the saturation in a solution particle by $\delta F(/ h)$ is sq $\delta F$ where, $q$ is the concentration-time amount of movement in solution and $s$ (Specific Movement Capacity) is a positive physical constant determined by material contained in the solution particle. Specific movement capacity is the movement per unit concentration-time amount of movement required to raise secondary saturation by $1 / h$.

- The rate at which saturation movement crosses a solution particle is proportional to concentration, time and secondary saturation gradient of solution particle. The following proposition is made that the amount of advective conductivity flux of solution particle $\left(\tilde{\kappa}\left(\frac{\mu \mathrm{g} \cdot \mathrm{h}}{\mathrm{ml}}\right)\right)$ is the difference between $\kappa_{\rho}$ (that amount of advective conductivity flux which is generated by movement density $\rho(x, t)$ (obtaining in the process of solution formation)) and $\kappa_{0}$ (an already existing constant (base) amount of advective conductivity flux (primarily obtaining in the independent of solute of $x$, solvent state)). The function of proportionality is called the amount of advective conductivity flux and is denoted by $\tilde{\kappa}=\kappa_{\rho}-\kappa_{0}$. The functions $\kappa_{\rho}$ and 
$\kappa_{0}$ are such that $\kappa_{\rho} \propto \rho(x, t)$ and $\kappa_{0} \propto \rho\left(y_{0}, t\right)$ where $y_{0}$ is the homogenous concentration $\operatorname{mix}$ in the base solvent without solute $x$, thus $\kappa_{\rho}=k \rho(x, t)$, $\kappa_{0}=\kappa \rho\left(y_{0}, t\right)$, and where $k, \kappa$ are constants of direct proportionality.

Considering an abitrary solution particle bridge $(A)$ (see Figure 1) the following relationship is proposed for its density dependent advective conductivity flux,

$$
\hat{\kappa}^{A}(x, t)=\frac{k \rho(x, t)}{x t}-\frac{\kappa \rho\left(y_{0}, t\right)}{y_{0} t} .
$$

In a homogenous mix, $\frac{\rho\left(y_{0}, t\right)}{y_{0} t}=\omega$. Thus, the advective conductivity flux [advective conductivity flux in the solvent of a solute of $x$, in the absence of the solute $x$ (before mixing)] is constant and Equation (1) reduces to,

$$
\hat{\kappa}^{A}(x, t)=\frac{k \rho(x, t)}{x t}-\kappa \omega .
$$

Assume that secondary saturation is increasing from left to right. The saturation gradient at the right end is $\frac{\partial F}{\partial x}(x+\delta x, t)$, so the rate at which movement crosses the right end is $-\hat{\kappa}^{A}(x, t) \frac{\partial F}{\partial x}(x+\delta x, t)$. Similarly, the rate at which movement crosses the left end is $-\hat{\kappa}^{A}(x, t) \frac{\partial F}{\partial x}(x, t)$. Since movement flows from regions of higher concentration to regions of lower concentration, movement thus should be entering the infinitesmal bridge through the right end and exiting the infinitesimal bridge through the left end. So in an infinitesimal time interval $(\delta t)$, the net amount of movement that enters the medium (solution particle bridge), is the amount that enters through the right end minus the amount that departs through the left end, which is given by,

$$
-\left(\hat{\kappa}^{A}(x, t) \frac{\partial F}{\partial x}(x+\delta x, t)-\hat{\kappa}^{A}(x, t) \frac{\partial F}{\partial x}(x, t)\right) \delta t .
$$

Considering a small time interval $(\delta t)$ the movement in the solution particle bridge changes by $\frac{\partial F}{\partial t}(x, t) \delta t$. The concentration-time amount of movement in the solution particle bridge is $\frac{\rho(x, t)}{x t} \delta x$. Thus by the first physical law, the net amount of movement

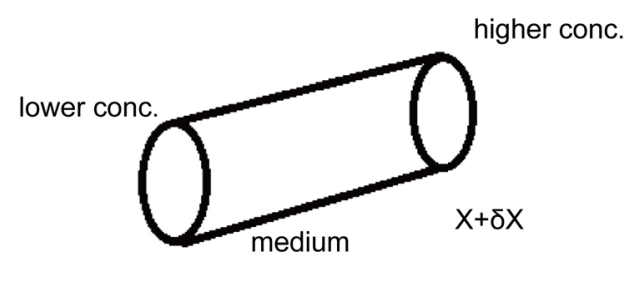

$x$

Figure 1. Infinitesmal bridge (medium) between solution particles at time $t$. 
which increase by $\frac{\partial F}{\partial t}(x, t) \delta t$ is $s \frac{\rho(x, t)}{x t} \delta x \frac{\partial F}{\partial t}(x, t) \delta t$. Assuming that the solution particle bridge is not generating or destroying movement itself. The following conclusion is made that it is equal to the amount of movement that entered the solution particle bridge in the interval time $\delta t$, that is

$$
s \frac{\rho(x, t)}{x t} \delta x \frac{\partial F}{\partial t}(x, t) \delta t=-\left(\hat{\kappa}^{A}(x, t) \frac{\partial F}{\partial x}(x+\delta x, t)-\hat{\kappa}^{A}(x, t) \frac{\partial F}{\partial x}(x, t)\right) \delta t .
$$

Dividing both sides by $\delta x \times \delta t$ and taking limits $\delta x, \delta t \rightarrow 0$ gives,

$$
s \frac{\rho(x, t)}{x t} \frac{\partial F}{\partial t}(x, t)=-\hat{\kappa}^{A}(x, t) \frac{\partial^{2} F}{\partial x^{2}}(x, t) .
$$

Further, division of Equation (5) both sides by,

$$
s \frac{\rho(x, t)}{x t} \text {. }
$$

The following result is immediate,

$$
\frac{\partial F}{\partial t}(x, t)=-\hat{\kappa}_{s}^{A}(x, t) \frac{\partial^{2} F}{\partial x^{2}}(x, t),
$$

where,

$$
\hat{\kappa}_{s}^{A}(x, t)=\frac{x t}{s \rho(x, t)} \hat{\kappa}^{A}(x, t)
$$

is the specific secondary saturation advective conductivity flux $(/ h)$ associated with a unit amount of movement in a solution bridge. This reduces to,

$$
\frac{\partial F}{\partial t}(x, t)=-\left(M-\rho_{I}^{\kappa, s}(x, t)\right) \frac{\partial^{2} F}{\partial x^{2}}(x, t),
$$

where,

$$
\rho_{I}^{\kappa, s}(x, t)=\frac{\kappa \omega x t}{s \rho(x, t)}=D(x, t) x t \text { and } M=\frac{k}{s} .
$$

The terms,

$$
\rho_{I}^{\kappa, s}(x, t), D(x, t)=\frac{\kappa \omega}{s \rho(x, t)} \text { and } M,
$$

are specific secondary saturation base-advection diffusivity flux $(/ h)$, secondary saturation advection diffusivity $\left(\frac{\mathrm{ml}}{\mu \mathrm{g} \cdot \mathrm{h}^{2}}\right)$, and specific secondary saturation initial or rest advection conductivity flux $(/ h)$ respectively.

The following relation from Equations (6) and (7) is established between advection conductivity flux and advection diffusivity flux,

$$
\overbrace{\hat{\kappa}_{s}^{A}(x, t)-M}^{\text {auxilliary advection conductivity flux }}=-\underbrace{\rho_{I}^{\kappa, s}(x, t),}_{\text {advection diffusivity flux }}
$$

where $M=\hat{\kappa}_{s}^{A}(0,0)$. 
It is important to note that $\hat{\kappa}_{s}^{A}(x, t)$ is the advection conductivity flux.

\section{Results}

\subsection{Applications to Deteministic Saturation Advection Kinetic Flow of Concentration of Efavirenz and Numerical Analysis}

There is use of the relationship developed from [6] [7] for some patient $P$. The first being the concentration-time profile of patient $P$ [7]. Additionally, the secondary saturation movement-concentration profile [6]. Separate projectiles are followed for secondary saturation using time and concentration for the single patient.

These two relations (Figure 2) are used to come up with the following fitted system of Equation(s) for patient $P$.

$$
F(x, t)=\left\{\begin{array}{l}
\lambda_{r}\left(\mathrm{e}^{-\lambda_{b} t}-\mathrm{e}^{-\lambda_{a} t}\right), t \in[0,24], \\
\frac{u x}{v+x}, \quad x \in\left[0, c_{\max }\right],
\end{array}\right.
$$

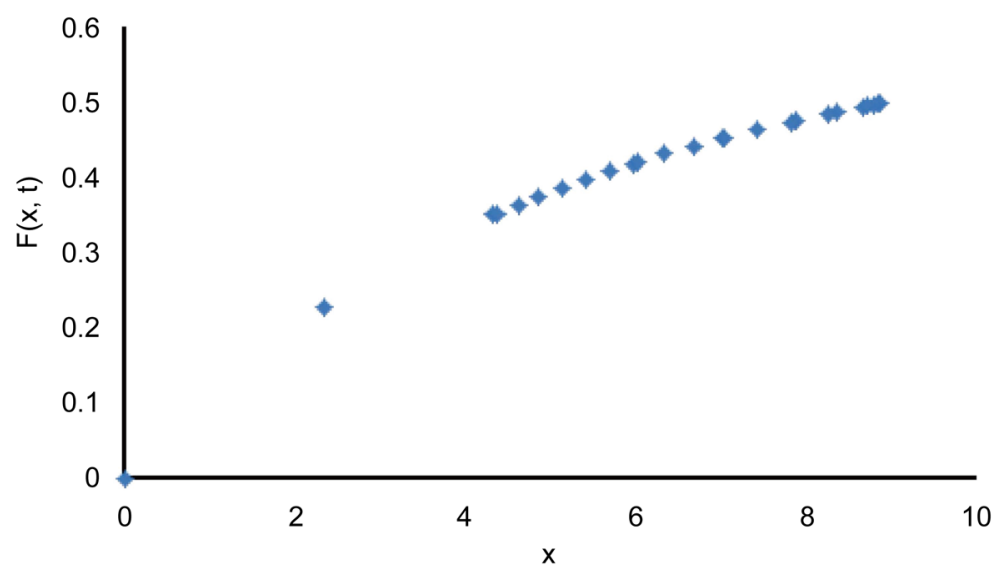

(a)

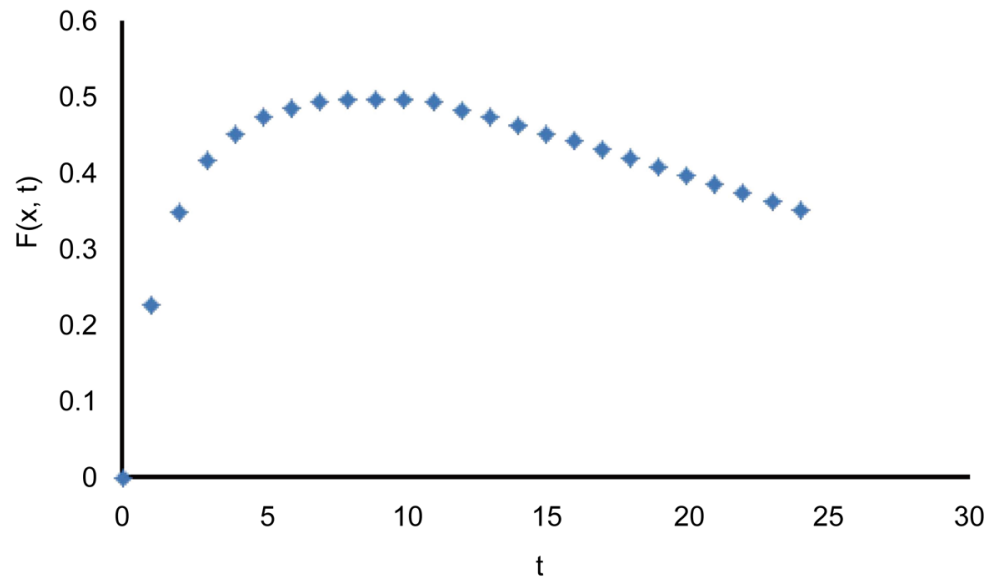

(b)

Figure 2. (a) The $x$-(concentration) projectile of $F(x, t)$ in patient $P$. (b)

The $t$-(time) projectile of $F(x, t)$ in patient $P$. 
where, $x$ is the concentration and $t$ is time. These two variables track solution particle dynamics. Furthermore, $\lambda_{r}, \lambda_{a}, \lambda_{b}, u$ and $v$ are constants to be found. The time (t) variable does not necessarily stop at 24 hours. This is only considered because it is the dosing interval of the drug efavirenz. Equation (9) estimates secondary saturation movement in terms of time and concentration independently (Table 1 and Table 2).

Following from Equation (9), Equation (6) assumes the form of,

$$
\frac{\mathrm{d} F}{\mathrm{~d} t}(x, t)=-\hat{\kappa}_{s}^{A}(x, t) \frac{\mathrm{d}^{2} F}{\mathrm{~d} x^{2}}(x, t),
$$

and Equation (8) becomes,

$$
\frac{\mathrm{d} F}{\mathrm{~d} t}(x, t)=-\left(M-\rho_{I}^{\kappa, s}(x, t)\right) \frac{\mathrm{d}^{2} F}{\mathrm{~d} x^{2}}(x, t) .
$$

The following conditions holds for secondary saturation advective diffusivity $D(x, t)$ at boundary points of loss/formation of the solution particle in the central volume space.

$$
\begin{aligned}
& \lim _{(x, t) \rightarrow(0,0)} D(x, t)=-\infty, \\
& \lim _{(x, t) \rightarrow(0,0)} \rho_{I}^{\kappa, s}(x, t)=0, \\
& \lim _{(x, t) \rightarrow(0, t)} D(x, t)=\infty \text { for some } t>0, \\
& \lim _{(x, t) \rightarrow(0, t)} \rho_{I}^{\kappa, s}(x, t)=M \text { for some } t>0 .
\end{aligned}
$$

It is noted that for $\hat{\kappa}_{s}^{A}(x, t)$ the following holds at boundary points of advective conductivity flux: $\hat{\kappa}_{s}^{A}(0,0):=M$, value at rest of saturation conductivity flux.

The part CC shows the advective diffusivity solution particle space (Central volume space) and CE shows the diffusivity in the complement space of the solution particle (neighbourhood volume space-Peripheral volume space) (Figure 3). A solution particle

Table 1. Parameter estimates in modelling saturation movement rate with respect to $t$ (Model 9(i)).

\begin{tabular}{ccccc}
\hline Parameters & Estimate & Std Error & $\mathrm{t}$ Value & $\operatorname{Pr}(>|t|)$ \\
\hline$\lambda_{r}$ & 0.623106 & 0.012214 & 51.02 & $<2 \times 10^{-16}$ \\
$\lambda_{b}$ & 0.022465 & 0.001203 & 18.68 & $5.35 \times 10^{-15}$ \\
$\lambda_{a}$ & 0.439258 & 0.017354 & 25.31 & $<2 \times 10^{-16}$ \\
\hline
\end{tabular}

Table 2. Parameter estimates in modelling saturation movement rate with respect to $x$ (Model 9(ii)).

\begin{tabular}{ccccc}
\hline Parameters & Estimate & Std Error & t Value & $\operatorname{Pr}(>|t|)$ \\
\hline$u$ & 0.801936 & 0.05934 & 135.1 & $<2 \times 10^{-16}$ \\
$V$ & 5.624198 & 0.126684 & 44.4 & $<2 \times 10^{-16}$ \\
\hline
\end{tabular}




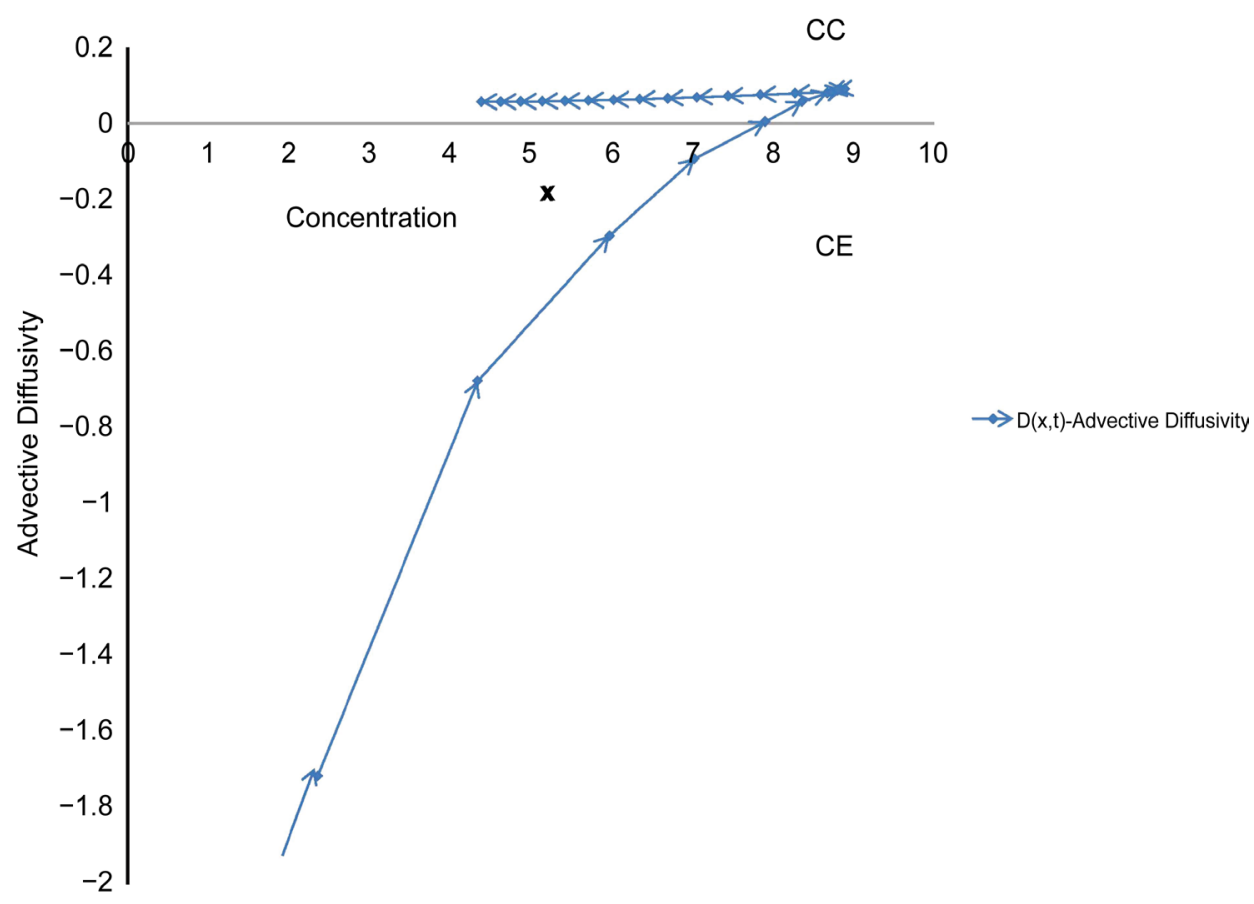

Figure 3. Time matched plot of advection diffusivity of saturation movement in the locality of two neighbouring volume spaces.

is affected by two neighbourhoods, one that is in the central volume space (CC) and the other is related to the complement (CE) immediate peripheral volume space. Movement in these two spaces varies according to the characterisation of these two spaces. The advective diffusivity flux's deceleration within the central compartment (that volume space which the solution particle is taken from) is given by $D_{C C}(x, t)$. While, the advective diffusivity flux's deceleration in the complement and is defined by $D_{C E}(x, t)$. They are equal only $D_{C C}(x, t)=D_{C E}(x, t)=0$. Thus,

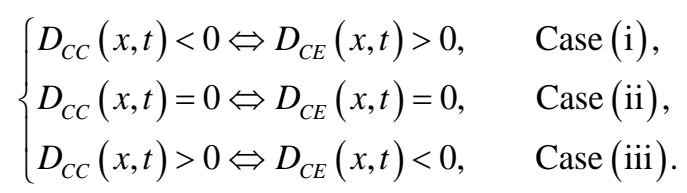

A positive value for advective diffusivity in the volume space (CC) implies that the movement is "concentrating" within CC space. However, a negative value shows movement is "concentrating" in the complement space (CE).

\subsection{Saturation Movement: Advection-Base Diffusivity and Conductivity Fluxes}

The diffusivity flux is an important parameter which is further investigated to see its dynamics in terms of the four entities that formed the basis of a solution particle. The existence of negative values for advection diffusivity shows presence of negative advective diffusivity flux (Figure 3). The following relations describe the expected advective diffusivity flux in the locality of the central volume space: 


$$
\begin{cases}D_{C C}(x, t)<\left.0 \Rightarrow \rho_{I}^{\kappa, s}(t)\right|_{C C}<0, & \text { Case (i), } \\ D_{C C}(x, t)=\left.0 \Rightarrow \rho_{I}^{\kappa, s}(t)\right|_{C C}=0, & \text { Case (ii), } \\ D_{C C}(x, t)>\left.0 \Rightarrow \rho_{I}^{\kappa, s}(t)\right|_{C C}>0, & \text { Case (iii), }\end{cases}
$$

where, $\left.\rho_{I}^{\kappa, s}\right|_{C C}$ is the advective diffusivity flux relative to the central volume space. Considering Equation (13), we deduce that for case (i), the advective diffusivity flux $\left(\left.\rho_{I}^{\kappa, s}\right|_{C C}\right)$ is in the direction of peripheral volume space from the central volume space. The advective diffusivity flux is zero for case (ii) the movement is inferred to be equal in both spaces, and in case (iii) advective diffusivity flux is positive implying movement is in the direction of central volume space from the peripheral volume space. The form of advection diffusivity flux has allowed for the postulation that diffusivity flux is actually a movement entity in equilibrium which comprises four entities. The principal parameter being the advection diffusivity flux. This is motivated by the same behaviour that has been observed in solution particle concentration in relation to saturation [6]. There is a mutualistic relationship postulated that enables us to study equilibrium of movement diffusivity flux. It is noted that the characterisation of saturation movement behaves similarly to concentration dynamics in-vivo. The reference movement with respect to saturation is advection, it is investigated and the constituent behaviour is suggested by Equation (14) (Table 3).

$$
\underbrace{\rho_{I}^{\kappa, s}(t)}_{\text {base-advection diffusivity flux }}=\overbrace{w t \mathrm{e}^{-n t}}^{\text {convection diffusivity flux }}+\underbrace{b\left(\mathrm{e}^{-d t}-1\right)}_{\text {passive diffusivity flux }}+\overbrace{\frac{f t}{g+t}}^{\text {saturation diffusivity flux }} \text {. }
$$

The characterisation of the four main diffusivity flux entities are shown and magnitudes of effects (Figure 4). The region CC represents that which affects the central volume space and $\mathrm{CE}$ that which affects the immediate peripheral volume space.

From Equation (8), we can infer that auxilliary conductivity flux consists of four main components and affects the movement into-solution particle and diffusivity flux affects

Table 3. Parameter estimates in modelling saturation movement diffusivity fluxes in Equation (14).

\begin{tabular}{ccccc}
\hline Parameters & Estimate & Std Error & t Value & $\operatorname{Pr}(>|t|)$ \\
\hline$W$ & -37.240855 & 1.320478 & -28.203 & $<2 \times 10^{-16}$ \\
$n$ & 0.416443 & 0.007034 & 59.207 & $<2 \times 10^{-16}$ \\
$b$ & 87.672322 & 12.141991 & 7.221 & $7.42 \times 10^{-7}$ \\
$d$ & 0.157279 & 0.002074 & 75.816 & $<2 \times 10^{-16}$ \\
$f$ & 99.406672 & 13.520844 & 7.352 & $5.74 \times 10^{-7}$ \\
$g$ & 1.979299 & 0.179995 & 10.996 & $1.12 \times 10^{-9}$ \\
\hline
\end{tabular}

$w$-residence rate of the convective diffusivity flux, $n$-declining rate of the convective diffusivity flux, $b$-maximum passive diffusivity flux rate constant, $d$-declining rate of the passive diffusivity flux, $f$-maximum saturation diffusivity flux rate constant and, $g$ - time at which the saturation diffusivity flux was half of $f$. 


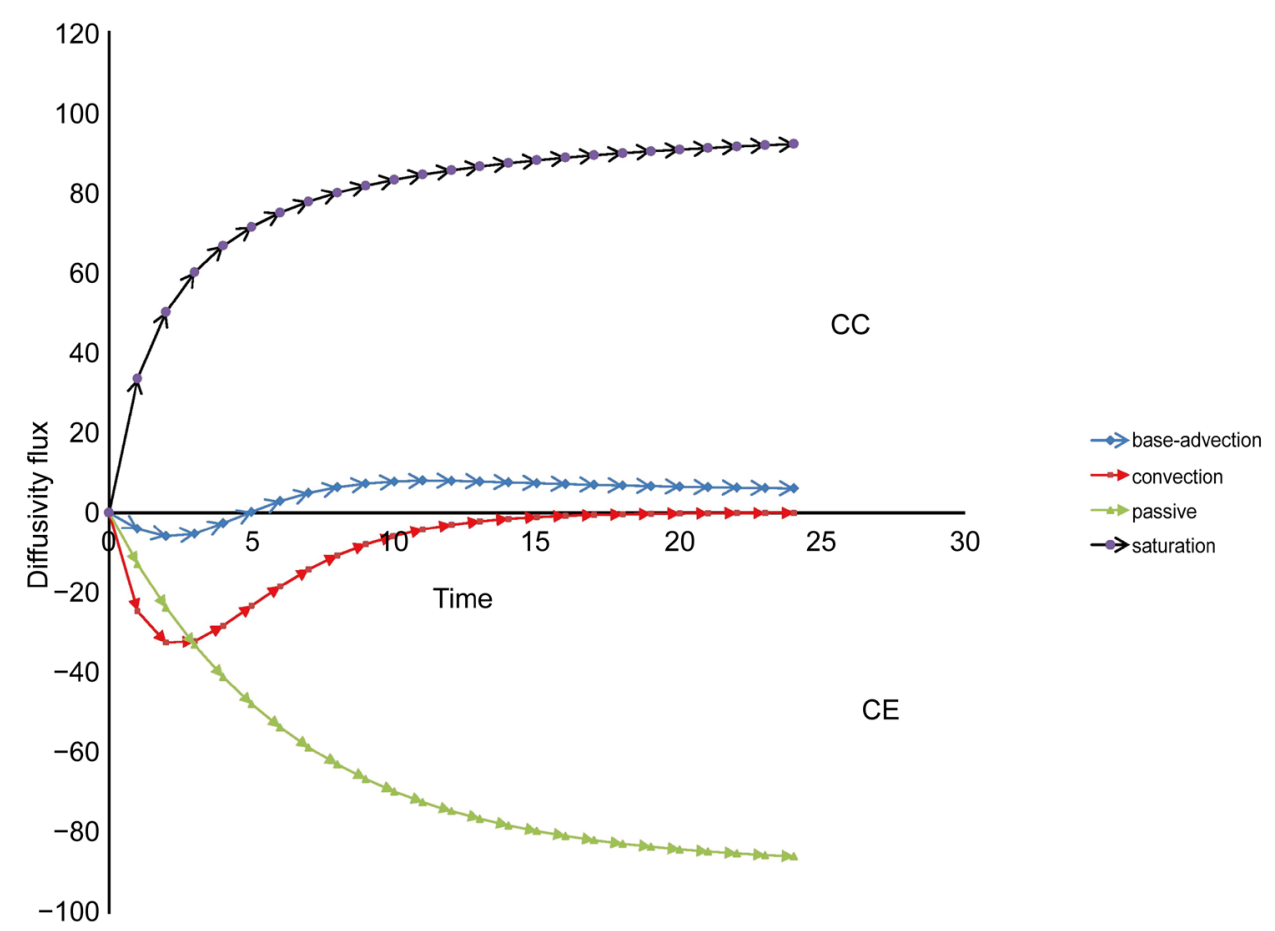

Figure 4. Components of diffusivity flux in the locality of two neighbouring volume spaces.

the movement out-of-solution particle. Diffusivity flux is symmetrical to conductivity flux in solution particle formation,

$$
\overbrace{\hat{\kappa}_{s}^{A}(x, t)-M}^{\text {auxilliary advection conductivity flux }}=\overbrace{-w t \mathrm{e}^{-n t}}^{\text {convection conductivity flux }}+\underbrace{-b\left(\mathrm{e}^{-d t}-1\right)}_{\text {passive conductivity flux }}+\overbrace{\frac{-f t}{g+t}}^{\text {saturation conductivity flux }}
$$

where, $w, n, b, d, f, g$ are constants with respect to conductivity flux as in Equation (14) and $M=5.12193$. It is noted that auxilliary conductivity flux is translated conductivity flux by a constant $(-M)$.

One notices a range of interpretations that can be deduced from the numerics done for the saturation dynamics of a solution particle (Figure 5). The advective conductivity flux is negatively correlated to advective diffusivity flux. Furthermore, auxilliary advective conductivity flux is negatively correlated to advective diffusivity flux thus advective diffusivity (see Equation (8)). The striking observation is how all the other variables relate similarly to the two variables that of concentration $(x)$ and saturation movement $(F(x, t))$ (Figure 5) ( $\operatorname{corr}(x, F(x, t))=0.95)$.

\section{Discussion}

This work proposes possible markers in the transportation of a drug which are conductivity and diffusivity. The partial differential equation derived has been shown to model the advection component of diffusivity and conductivity.

A new development is inferred where the movement diffusivity flux is shown to be a function of four primary components. The primary components have been identified as 


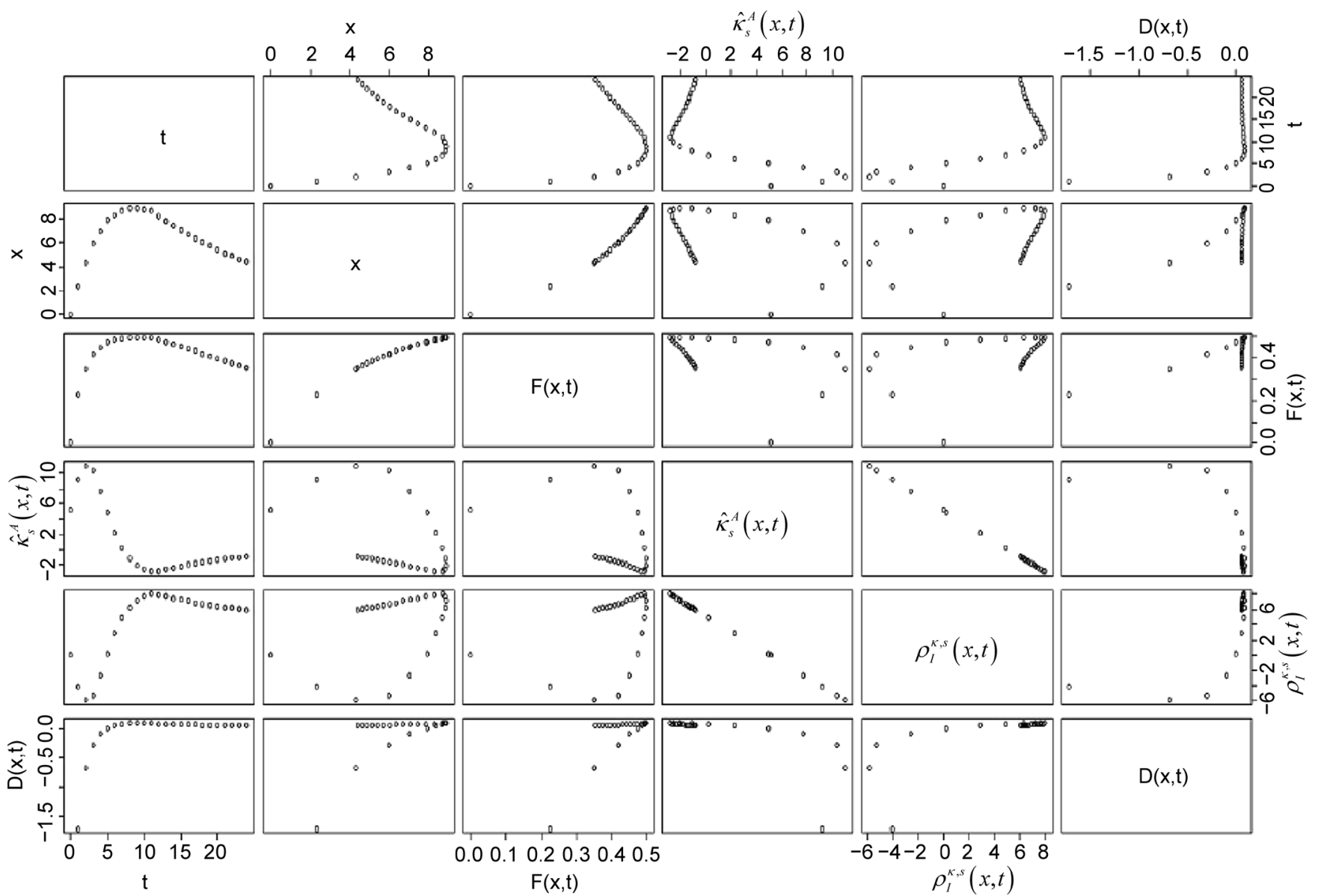

Figure 5. Summary scatter time-matched plot of the relationship between the following variables $t, x, F(x, t), \hat{\kappa}_{s}^{A}(x, t), \rho_{I}^{\kappa, s}(x, t)$ and $D(x, t)$ for patient $P$ informed by Equations (9), (10) and (11).

advection, saturation, convection and passive. The total movement diffusivity flux in the system is zero for any given concentration at a given time. The two movement diffusivity fluxes convection and passive are inferred to exert their influence in the peripheral volume system. However, saturation and advection predominantly exerts influence in the central volume space. The increase in advective diffusivity flux shows a corresponding reduction of advective conductivity, this is postulated from the negative correlation that these two parameters share in this system. It has been shown that, not only concentration affect diffusivity and conductivity, but also the state of the neighbourhood. Additionally, there are potential factors that could be involved in the processes of transportation, absorption and elimination of the drug.

The advective diffusivity of the drug has partly negative values. Furthermore, it is not a constant and the regions of negative advective diffusivity are relatively very high as compared to positive diffusivity in the volume spaces. Initially, the negative values of advective diffusivity indicate that the peripheral volume space has relatively higher levels of flow. The drug is flowing more in the peripheral space. Thus the drug is quickly available to the peripheral space initially and furthermore is "concentrating" in this 
space [8]. This could potentially have beneficial or negative effects depending on what is being transported and the individual's reaction to it. This result led to the proposal of the potential effect on CNS adverse drug reactions. There is increased transportation in the peripheral regions in the initial stages. Other researchers have found that the majority of efavirenz-induced CNS adverse drug reactions appear early even after a single dose [9]. Diffusivity is one parameter that could potentially be affected with the presence of the residual drug in the system (from previous dose) in the volume spaces. This work shows that transportation consistently alters in the human body and there are differences in the two neighbouring volume spaces. A relatively controlled state is achieved during the terminal phase. The solution particle is "concentrating" in the central volume space.

Inference on in-vivo release can be made using models developed. The interpretation of kinetic conductivity developed here allowed study of diffusivity. The work made use of data informed directly from the developed PK/PD models [6] [7]. Other researchers have proposed models to deal with complications involving concentration, time and location dependence on diffusivity. As a result, Fick's formalisation has been thought to be less effective [10]. However, this work recast Fick's law with the aid of secondary saturation transportation that has been found to be positively correlated to concentration. In place of the location variable it uses concentration and retains the time variable. This system enables the study of characterisation of diffusion and conduction. It proposes a new space that can be used to further our understanding of the phenomenological approach of the Fick's law [11].

Diffusivity and conductivity of drugs in patients finds its use in studying drug release kinetics. This is important to the successful design of polymeric delivery systems bearing in mind that in-vivo release models are laborious and costly [12]. Using the new variable space proposed experiments can be developed to aid our understanding of diffusion across a range of applications [4] [11]. The results proposed here have implications in the way gradient induced diffusion could be handled mathematically. Currently, diffusivity and conductivity are investigated using the location and time as independent variables. This work proposes a different approach in investigating them in-vivo.

Diffusivity is symmetrical to conductivity in solution particle formation. These two processes occur concurrently. The pro-gradient-driven-movement entities of a solution particle with respect to conductivity and diffusivity are convective and passive. While, anti-gradient-driven-movement entities are saturation and advection. This analysis is inferred from the characterisation of a solution particle in Nemaura (2015) [6]. The derivation of the phenomenon observed, points to advection as a significant primary entity.

With the aid of the characterisation of a saturation kinetic flow (strictly positively correlated relation to concentration), one can use this to infer the behaviour of the solution particle in relation to its neghbourhood through advective diffusivity. This work shows the existence of a model which is deterministic and describes the behaviour of how a solution particle gain/lose movement relative to its local neighbourhood. The 
gain of movement is synonymous with conductivity and loss of movement is synonymous with diffusivity. The two advection movement fluxes are symmetric about half the initial value of advection conductivity $(M / 2)$ and also all the other subsequent corresponding components in the $24 \mathrm{~h}$.

\section{Acknowledgements}

The author would like to thank the following; C. Nhachi, C. Masimirembwa, and G. Kadzirange, AIBST and The College of Health Sciences, University of Zimbabwe.

\section{References}

[1] Mehrer, H. and Stolwijk, N.A. (2009) Heroes and Highlights in the History of Diffusion. Diffusion Fundamentals, 11, 1-32.

[2] Narasimhan, T.N. (1999) Fourier's Heat Conduction Equation: History, Influence and Connections. Reviews of Geophysics, 37, 151-172. http://dx.doi.org/10.1029/1998RG900006

[3] Feldman, J. (2007) The Heat Equation (One Space Dimension). http://www.math.ubc.ca/feldman/m267/heat1d.pdf

[4] Crank, J. (1975) The Mathematics of Diffusion. 2nd Edition, Oxford University Press, Oxford.

[5] De Kee, D., Liu, Q. and Hinestroza, J. (2005) Viscoelastic (Non-Fickian) Diffusion. The Canadian Journal of Chemical Engineering, 83, 913-929. http://dx.doi.org/10.1002/cjce.5450830601

[6] Nemaura, T. (2015) Modeling Transportation of Efavirenz: Inference on Possibility of Mixed Modes of Transportation and Kinetic Solubility. Frontiers in Pharmacology http://dx.doi.org/10.3389/fphar.2015.00121

[7] Nemaura, T. (2014) Projections of Pharmacokinetic Parameter Estimates from Middose Plasma Concentrations in Individuals on Efavirenz; a Novel Approach. African Journal of Pharmacy and Pharmacology, 8, 929-952.

[8] Argyrakis, P., Chumak, A.A., Maragakis, M. and Tsakiris, N. (2009) Negative Diffusion Coefficient in a Two-Dimensional Lattice-Gas System with Attractive Nearest-Neighbor Interactions. Physical Review B, 80, 1-7. http://dx.doi.org/10.1103/PhysRevB.80.104203

[9] Apostolova, N., Funes, H.A., Blas-Garcia, A., Galindo, M.J., Alvarez, A. and Esplugues, J.V. (2015) Efavirenz and the CNS: What We Already Know and Questions That Need to Be Answered. Journal of Antimicrobial Chemotherapy, 70, 2693-708. http://dx.doi.org/10.1093/jac/dkv183

[10] Petropoulos, J.H., Sanopoulou, M. and Papadokostaki, K.G. (2009) Beyond Fick: How Best to Deal with Non-Fickian Behavior in a Fickian Spirit. Diffusion Fundamentals, 11, 1-21.

[11] Philibert, J. (2005) One and a Half Century of Diffusion: Fick, Einstein, Before and Beyond. Diffusion Fundamentals, 2, 1-10.

[12] Fu, Y. and Kao, W. J. (2010) Drug Release Kinetics and Transport Mechanisms of NonDegradable and Degradable Polymeric Delivery Systems. Expert Opinion on Drug Delivery, 7, 429-444. http://dx.doi.org/10.1517/17425241003602259 
Submit or recommend next manuscript to SCIRP and we will provide best service for you:

Accepting pre-submission inquiries through Email, Facebook, LinkedIn, Twitter, etc. A wide selection of journals (inclusive of 9 subjects, more than 200 journals)

Providing 24-hour high-quality service

User-friendly online submission system

Fair and swift peer-review system

Efficient typesetting and proofreading procedure

Display of the result of downloads and visits, as well as the number of cited articles

Maximum dissemination of your research work

Submit your manuscript at: http://papersubmission.scirp.org/

Or contact jamp@scirp.org 